\title{
NONLINEAR UPCONVERSION-RATE IN Er-DOPED FIBERS
}

\author{
B. Jaskorzyńska* ${ }^{*}$ S. SergeyeV ${ }^{\dagger}$, M. Świllo and D. Khoptyar
}

Royal Institute of Technology, Dept. of Electronics, Electrum 229, 16440 Kista, Sweden

\begin{abstract}
Energy transfer between excited rare-earth ions has been widely used for realizing upconversion lasers and also recognized as a gain limiting factor in high-concentration Er-doped amplifiers. The energy transfer leads to upconversion of the excitation which for randomly distributed (not clustered) ions is called homogeneous upconversion. It was commonly assumed that the rate of homogeneous upconversion is a linear function of the population inversion $\mathrm{N}_{2}$. However, recently published Monte Carlo simulations predict that the homogeneous upconversion rate is a nonlinear function of $\mathrm{N}_{2}$ and that it, moreover, depends on the pump and signals rates. In this paper we review some of our experimental results confirming those predictions. We also propose a statistical, analytical model describing the observed homogeneous upconversion behavior in Er-doped fibers.
\end{abstract}

PACS numbers: 78.20.Bh, 78.55.-m, 42.55.Rz

\section{Introduction}

Interactions between excited Er-ions are well known to reduce gain in high-concentration Er-doped amplifiers. Excitation energy transferred from one ion (donor) to another (acceptor) causes de-excitation of the donor and higher excitation (upconversion) of the acceptor. For randomly distributed (not clustered) ions this process is called homogeneous upconversion (HUC). The upconverted ion typically relaxes back to the metastable level losing the excess energy to phonons.

The earlier models describing the upconversion in Er-doped fiber amplifiers commonly assumed a uniform (equidistant) distribution of excited ions, and hence a linear dependence of the upconversion rate on the population inversion $\mathrm{N}_{2}[1,2]$. The observed nonlinear behavior at high pumping rates (high population inversion) was attributed to the contribution from ultra fast upconversion among clustered ions $[1,2]$. The ensemble of all the ions was divided into two sub-ensembles:

* corresponding author; e-mail: bj@ele.kth.se

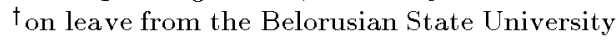


the clustered ions and the ions which are randomly distributed. It was further assumed that the excitation migration is much faster than the upconversion (the kinetic limit) and therefore, the excitation distribution for the random sub-ensemble is smoothed out. In Ref. [3] an alternative, statistical approach has been proposed taking into account a finite migration rate. The results obtained by Monte Carlo simulations showed that even for randomly distributed Er ions the $\mathrm{HUC}$ rate $W_{\mathrm{c}}$ is a nonlinear function of $\mathrm{N}_{2}$, and that it is accelerated by the migration process. It was also predicted that a similar HUC enhancement should take place when the pump and the signal powers are increased while $\mathrm{N}_{2}$ remains unchanged. The nonlinearity of $W_{c}$ was earlier predicted for dye molecules statistically uniformly distributed in solution [4].

In order to verify those predictions we have performed several experiments based on decay measurements for the metastable (at $1.5 \mu \mathrm{m}$ ) and the upconverted (at $980 \mathrm{~nm}$ ) fluorescence. The experiments were designed in such a way that the contribution from possible excess clustering was separated and only the upconversion of the randomly distributed ions was analyzed. The results [5-7] clearly confirm that the HUC rate becomes strongly nonlinear for high population inversions, and that it depends not only on the population inversion but also on the level of the pump and signal rates applied to reach a given inversion.

Although the theory of Ref. [3] motivated our experimental investigations, the very time consuming Monte Carlo simulations make the method unpractical as a tool for device design or as an interactive feedback to experimental studies. Moreover, the approximations made in the theoretical formulation are not clearly justified. We propose an analytical model for the upconversion in Er-doped waveguides. It is based on well established theories [8] with a known maximum error due to the involved approximations. The main assumption made is that the migration is significantly faster than the upconversion, which is well justified for realistic Er-doped devices. The solution is readily obtained by solving one transcendental equation. The model reconstructs all the new features predicted in [3]. We believe that it will serve as a useful tool for investigating and design of Er-doped devices, especially for planar, integrated amplifiers or short, heavily doped fiber lasers where the upconversion plays a significant role.

\section{Theoretical model}

The starting point for the proposed here model is the following set of the local rate equations for a quasi-two level system:

$$
\frac{\mathrm{d} n_{k}}{\mathrm{~d} t}=\left(1-\beta n_{k}\right) \alpha-n_{k}-n_{k} \sum_{l \neq k} P_{k l}-n_{k} \sum_{j \neq k} W_{k j}+\sum_{j \neq k} W_{k j} n_{j} .
$$

Here time $t$ is normalized with respect to a lifetime $\tau_{2}$ of the second excited level, $n_{k}$ is the probability of localization of an excitation at the ion with a number $k$, $\alpha=\sigma_{\mathrm{a}} I$ with $I$ standing for a pump rate, and $\beta=\left(\sigma_{\mathrm{a}}+\sigma_{\mathrm{e}}\right) / \sigma_{\mathrm{a}}$, where $\sigma_{\mathrm{a}}$ and $\sigma_{\mathrm{e}}$ are the absorption and emission cross-section, respectively, at the pump wavelength.

The rates of upconversion, $P_{k l}$, and migration, $W_{k j}$, are expressed using the Förster-Dexter theory $[9,10]$ for the dipole-dipole mechanism of an energy 
transfer

$$
P_{k l}=\frac{R_{\mathrm{up}}^{6}}{R_{k l}^{6}}, \quad W_{k j}=\frac{R_{\mathrm{m}}^{6}}{R_{k j}^{6}},
$$

where $R_{k l}$ is a distance between excited ions $(k)$ and $(l) ; R_{k j}$ is a distance between ions $(k)$ and $(j), R_{\mathrm{up}}, R_{\mathrm{m}}$ are critical distances for the upconversion and migration processes $[3,4,8-15]$, respectively.

After formal averaging of Eqs. (1) over all the ions the global upconversion rate is expressed as

$$
W_{\mathrm{c}}=\lim _{N_{0} \rightarrow \infty} \frac{\sum_{k=1}^{N_{0}} n_{k} \sum_{l \neq k} P_{k l}}{\sum_{k=1}^{N_{0}} n_{k}}=-\frac{\mathrm{d} n}{\mathrm{~d} t} \frac{1}{n}+\frac{\alpha(1-\beta n)}{n}-1,
$$

where $n=\lim _{N_{0} \rightarrow \infty} \frac{1}{N_{0}} \sum_{k=1}^{N_{0}} n_{k}$ is a population inversion level and $N_{0}$ is a number of ions.

In order to effectively perform the averaging required for calculating the population inversion we assume $\sum_{j \neq k} W_{k j} n_{j} \approx n \sum_{j \neq k} W_{k j}$, which is a good approximation $[4,11]$ for the dipole-dipole mechanism of energy transfer. We further restrict our considerations to the case when the ratio of the migration to quenching critical distances is $\left(R_{\mathrm{m}} / R_{q}\right)^{6} \gg 1$, which is well justified for Er-doped glass [16]. This enables us to treat all the ions (acceptors) and excited ions (donors) as two independent ensembles within the frame of the hopping mechanism [8], and to express Eqs. (1) as

$$
\frac{\mathrm{d} n\left(S_{1}, S_{2}\right)}{\mathrm{d} t}=\left[1-\beta n\left(S_{1}, S_{2}\right)\right] \alpha-n\left(S_{1}, S_{2}\right)\left(1+S_{1}\right)-\left[n\left(S_{1}, S_{2}\right)-n\right] S_{2},
$$

where $n=\int_{0}^{\infty} \int_{0}^{\infty} n\left(S_{1}, S_{2}\right) f\left(S_{1}\right) f\left(S_{2}\right) \mathrm{d} S_{1} \mathrm{~d} S_{2}, S_{1}=\sum_{l \neq k} P_{k l}, S_{2}=\sum_{j \neq k} W_{k j}$ are independent stochastic variables with the distributions $[4,11,13]$

$$
f\left(S_{i}\right)=\frac{k_{i}}{2 \sqrt{\pi} S_{i}^{3 / 2}} \exp \left(-\frac{k_{i}^{2}}{4 S_{i}}\right), \quad i=1,2,
$$

where $k_{1}=\sqrt{\pi} \gamma n, k_{2}=\sqrt{\pi r / 2} \gamma, \gamma=c_{\mathrm{Er}} / c_{\mathrm{up}}, c_{\mathrm{Er}}$ is the concentration of Er ions, $c_{\mathrm{up}}=\left[(4 \pi / 3) R_{\mathrm{up}}^{3}\right]^{-1}$ is the critical concentration for the upconversion, $r=\left(R_{\mathrm{m}} / R_{\mathrm{up}}\right)^{6}$. The factor $1 / \sqrt{2}$ is the Huber correction [14] accounting for the migration reversibility, $k_{1}$ and $k_{2}$ are proportional to the concentration of excited and all ions, respectively.

Setting $\mathrm{d} n / \mathrm{d} t=0$ and averaging the rate equation (4) over all donors and all acceptors with the use of the distribution functions (5) we arrive at the following transcendental equation for the population inversion in the steady-state case

$$
n=\frac{\alpha(n+\sqrt{r / 2})}{1+\beta \alpha} \frac{F(k(n+\sqrt{r / 2}) / 2 \sqrt{1+\beta \alpha})}{n+\sqrt{r / 2} F(k(n+\sqrt{r / 2}) / 2 \sqrt{1+\beta \alpha})},
$$

with $F(x)=1-\sqrt{\pi} x \exp \left(x^{2}\right) \operatorname{erfc}(x), k=\sqrt{\pi} \gamma$.

Using the asymptotic form of $F(x) \approx 1 / 2 x^{2}(x \rightarrow \infty)$ and assuming high ion concentrations $(k \sqrt{r} \gg 1)$ we also obtain the following linear asymptote for the upconversion rate at small pumping rates, $\alpha \rightarrow 0(n \rightarrow 0)$,

$$
W_{\mathrm{c}}=\frac{\pi c_{\mathrm{Er}}^{2} n \sqrt{r}}{2 \sqrt{2}}\left(\frac{4 \pi}{3}\right)^{2} R_{\mathrm{up}}^{6}
$$




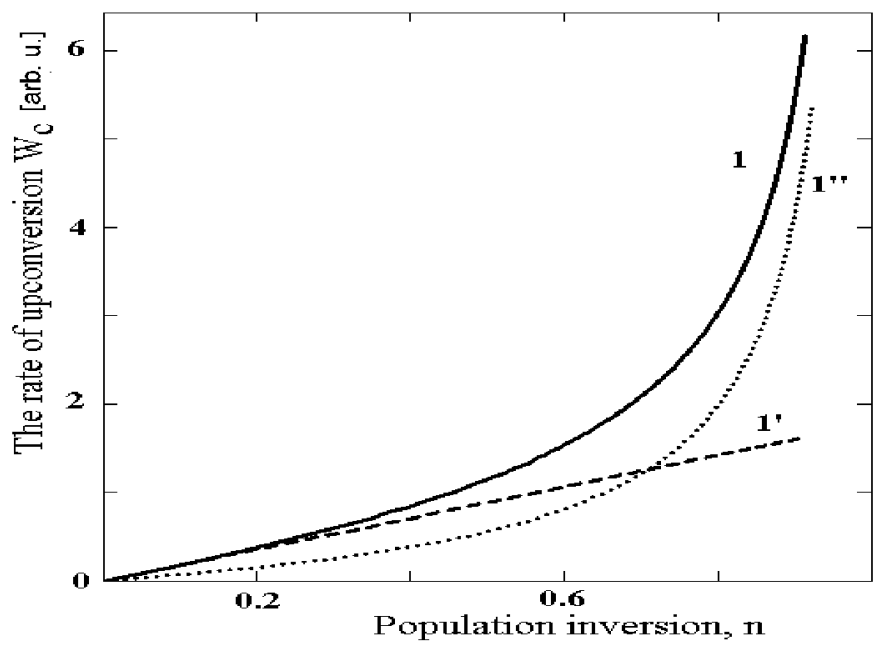

Fig. 1. The calculated rate of upconversion vs. population inversion: 1 - with migration, $1^{\prime}$ - without migration, $1^{\prime \prime}$ - the asymptote for small pumping rates.

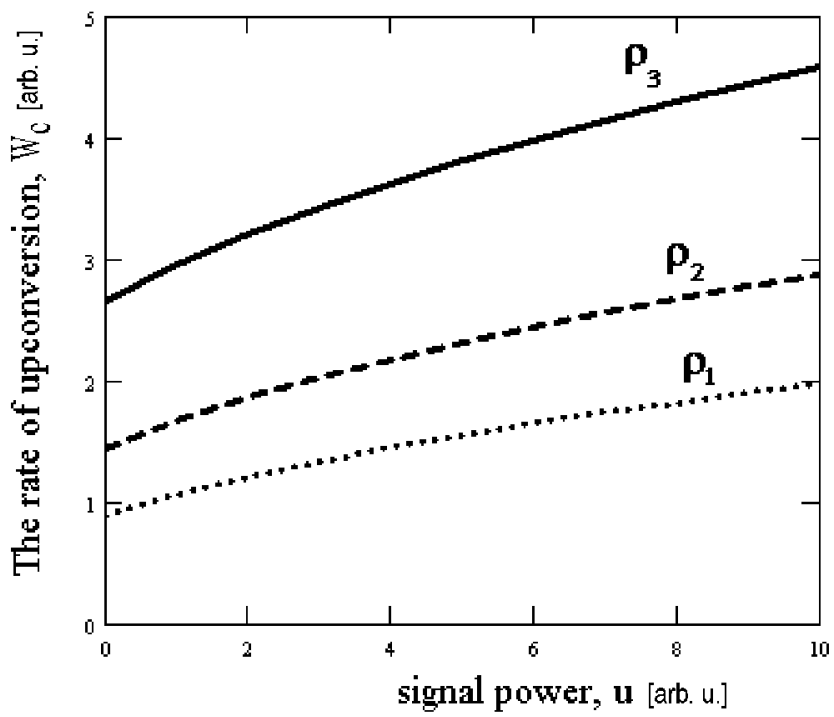

Fig. 2. The calculated rate of upconversion vs. signal power for three different Er-concentration $\rho: \rho_{1}<\rho_{2}<\rho_{3}$. The pump power is adjusted so that the population inversion remains constant.

In Fig. 1 we plotted the upconversion rate as a function of the population inversion. The nonlinear characteristic (curve 1) with a linear asymptote (curve $1^{\prime}$ ) for small population inversions are consistent with the results of the experiments $[1,2,5,6,16]$ and the simulations [3]. Also enhancement of the upconversion caused by migration predicted in [3] is clearly seen from our model (curves $\left.1,1^{\prime \prime}\right)$. 
In the regions where the ion density is highest the upconversion rate (strongly dependent on the ion separation) is much faster than realistic pumping rates. This results in spatial hole burning in the excitation distribution. In the absence of migration the fastest upconverting regions would not any longer efficiently contribute to the average upconversion. Migration fills up the holes and hence it accelerates the upconversion process.

In Fig. 2 we show that the upconversion rate at the fixed population inversion depends on the signal power (the pump power is adjusted to keep the inversion at the fixed level). This peculiar behavior, first predicted in [3], was also confirmed by our experiments [5-7], as it is shown in the next section. It can be understood by noticing that at increased pump powers the holes burnt by the upconversion are filled up more efficiently and therefore, similar to the impact of migration, the average upconversion rate is enhanced.

\section{Experimental results}

In order to investigate the behavior of the upconversion characteristics we performed a series of the decay measurements for the metastable $(1.5 \mu \mathrm{m})$ and the upconverted $(980 \mathrm{~nm})$ fluorescence transverse to fibers densely doped with erbium. The method that we developed to determine the upconversion rate in absolute units is described in [5].

In Fig. 3 we show a clearly nonlinear behavior of the upconversion rate as a function of the population inversion.

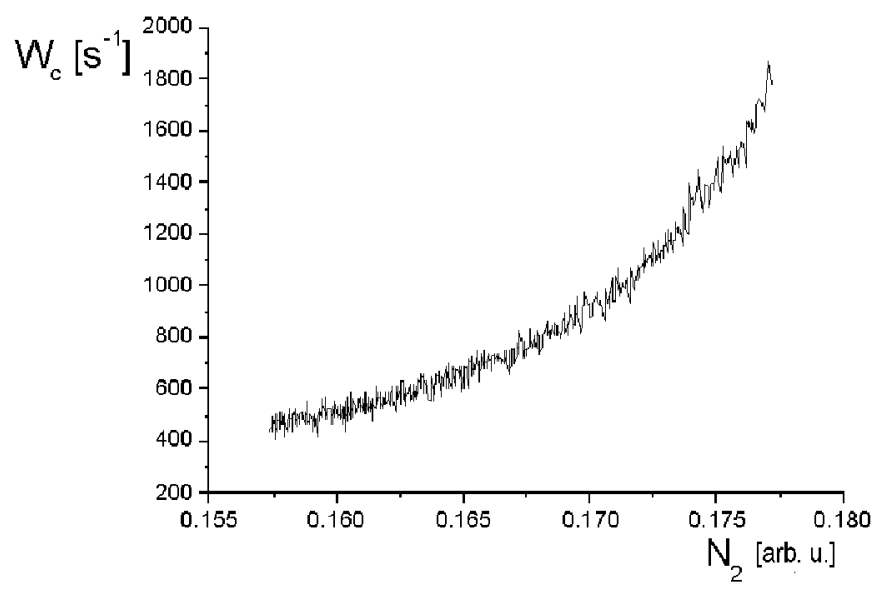

Fig. 3. The measured upconversion rate vs. population inversion.

Three decay curves for the $1.5 \mu \mathrm{m}$ fluorescence from the same steady-state level of the population inversion but obtained with different pump-signal powers are shown in Fig. 4. The higher the pump-signal powers the larger the upconversion rate. The upconversion rate at $t=0$ was determined as $W_{\mathrm{c}}(0)=$ $-\mathrm{d} / \mathrm{d} t_{t=0}(\ln n)+1 / \tau_{2}$. The higher slope corresponds to a larger $W_{c}$. The resulting 


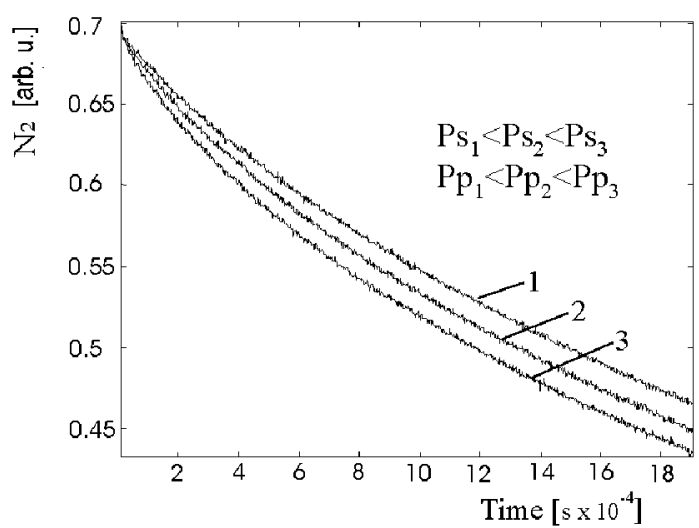

Fig. 4. The measured decay curves from the same level of steady-state population inversion but obtained with different pump and signal powers. The slopes increase with increasing pump-signal powers.

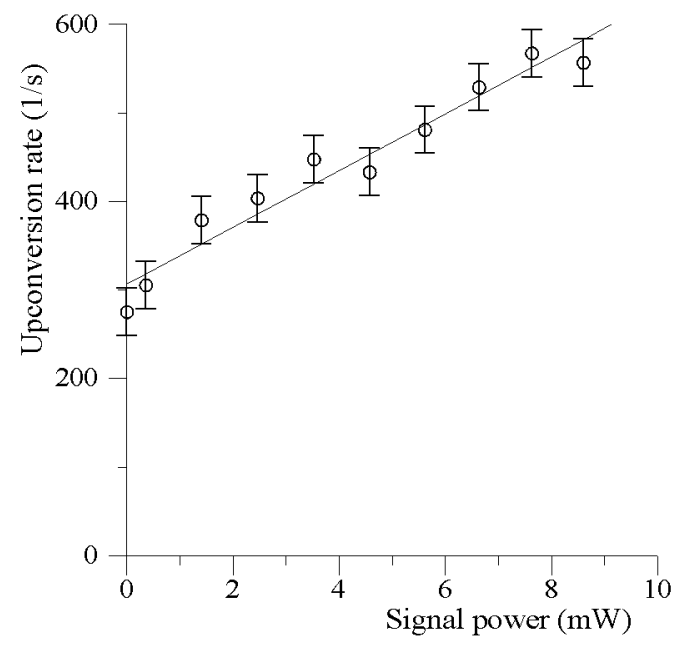

Fig. 5. The upconversion rate vs. signal power determined from the measured decay curves as those shown in Fig. 4.

$W_{\mathrm{c}}(0)$ for 10 different pump-signal powers are shown in Fig. 5. The almost linear dependence is in good agreement with the calculated curves of Fig. 2. Time resolution for our measurements was about $2 \mu \mathrm{s}$ which implies that the contribution from much faster decaying $(<50 \mathrm{~ns}$ ), possible Er-clusters, did not influence the presented results.

\section{Summary}

We proposed a statistical model for description of migration assisted upconversion in Er-doped glasses. The model provides an analytical solution for the population inversion and the upconversion rate. It well reproduces all the features 
predicted by the earlier published Monte Carlo simulations for Er-doped fibers. We also reviewed the results of our experiments that provided the first experimental evidence of the nonlinear behavior of the upconversion rate on the population inversion, as well as the first confirmation of the theoretical predictions that for a fixed population inversion the upconversion rate also depends on the pump and signal powers.

\section{Acknowledgments}

The Swedish Foundation for Strategic Research (SSF) and the Swedish Research Council for Engineering Sciences (TFR) are gratefully acknowledged for supporting this research.

\section{References}

[1] R.S. Quimby, W.J. Miniscalo, B. Thompson, J. Appl. Phys. 76, 4472 (1994).

[2] J. Nilsson, P. Blixt, B. Jaskorzynska, J. Babonas, J. Lightwave Technol. 13, 341 (1995).

[3] J.L. Philipsen, A. Bjarklev, IEEE J. Quantum Electron. 33, 845 (1997).

[4] V.A. Gaisenok, A.I. Slobodyanyuk, Opt. Spektrosk. 65, 39 (1988).

[5] M. Swillo, D. Bremberg, B. Jaskorzynska, S. Helmfrid, J.L. Philipsen, in: Integrated Photonic Research, OSA Technical Digest, Optical Society of America, Washington (DC) 1999, p. 73.

[6] J.L. Philipsen, J. Broeng, A. Bjarklev, S. Helmfrid, D. Bremberg, B. Jaskorzynska, B. Palsdottir, IEEE J. Quantum Electron. 36, 1741 (1999).

[7] D. Bremberg, S. Helmfrid, B. Jaskorzynska, M. Swillo, J.L. Philipsen, B. Pálsdóttir, Electron. Lett. 14, 1189 (1999).

[8] E.N. Bodunov, Opt. Spektrosc. 74, 311 (1993).

[9] T. Förster, Ann. Phys. 2, 55 (1948).

[10] D.L. Dexter, J. Chem. Phys. 21, 836 (1953).

[11] A.I. Slobodyanyuk, Ph.D. Thesis, Belarusian State University, Minsk 1988.

[12] B.E. Vugmeister, Phys. Status Solidi B 76, 161 (1976).

[13] N.M. Rozman, Opt. Spektrosk. 4, 536 (1958) [Opt. Spectrosc. (USSR) 4, 202 (1958)].

[14] D.L. Huber, D.S. Hamilton, B. Barnet, Phys. Rev. B 16, 4642 (1977).

[15] A.I. Burstein, Sov. Phys. Usp. 27, 579 (1984).

[16] M. Hempstead, C.C. Ye, J.S. Wilkinson, P. Camy, P. Laborde, C. Lerminiaux, in: Proc. 7th European Conf. on Integrated Optics, Vol. 1, Delft University Press, Delft 1995, p. 233. 